\begin{abstract}
¿Cómo citar este artículo?
Martínez-Castro, C. A., Zapata-Cardona, L. y Castro, W. F. (enero-abril, 2020). ¿Qué dicen los profesores que enseñan matemáticas sobre las bondades y limitaciones de la evaluación docente? Revista Virtual Universidad Católica del Norte, (59), 71-90. doi: https://doi.org/10.35575/rvucn.n59a5
\end{abstract}

\title{
¿Qué dicen los profesores que enseñan matemáticas sobre las bondades y limitaciones de la evaluación docente?
}

What do mathematics teachers say about the bounties and limitations of teacher evaluation?

\author{
Cindy Alejandra Martínez-Castro \\ Licenciada en Educación Básica con énfasis en \\ Matemáticas \\ Universidad de Antioquia \\ Colombia \\ cindy.martinez@udea.edu.co \\ Orcid: https:/ /orcid.org/0000-0001-6846-0693
}

\author{
Lucía Zapata-Cardona \\ Doctora en Educación Matemática \\ Universidad de Antioquia \\ Colombia \\ lucia.zapata1@udea.edu.co \\ Orcid: https://orcid.org/0000-0003-4266-5273
}

\section{Walter F. Castro}

Doctor en Didáctica de las Matemáticas

Universidad de Antioquia

Colombia

walter.castro@udea.edu.co

Orcid: https://orcid.org/0000-0002-7890-681X

Recibido: : 05 de julio de 2019 Evaluado: 16 de septiembre de 2019

Aprobado: 15 de noviembre de 2019

Tipo de artículo: Investigación científica y tecnológica.

\section{Resumen}

Estudiar la valoración que los profesores que enseñan matemáticas hacen sobre la evaluación docente es esencial para apoyar la reorganización de este proceso. En este artículo se estudian las bondades y limitaciones que los profesores en la educación obligatoria perciben sobre la evaluación docente. La metodología atendió a un paradigma cualitativo, donde los informantes fueron 128 profesores de instituciones educativas, de carácter público, de las regiones del departamento de Antioquia, Colombia. Los profesores enseñaban en diferentes niveles del sistema educativo, y respondieron a una encuesta que indagaba sobre las bondades y limitaciones del sistema de evaluación actual. Los resultados muestran que los profesores ven muchas más limitaciones que bondades en el sistema de evaluación docente, a pesar de reconocer su importancia. Entre las limitaciones señaladas se encuentran la subjetividad del proceso, la poca retroalimentación para los docentes y la poca pertinencia. Dentro de los resultados se destaca que el sistema de evaluación docente debería tener en cuenta la formación del profesor, la producción académica, y el contexto en el cual se desempeña. Se concluye de este estudio que es necesario transformar la mirada de la evaluación sumativa a una evaluación formativa que aporte al mejoramiento de la labor docente.

Palabras clave: Desarrollo profesional, Evaluación docente, Evaluación formativa, Percepciones de profesores, Profesores de matemáticas. 


\section{| Abstract}

Studying the assessment that mathematics teachers make on teacher evaluation is essential to support the reorganization of this process. This paper studies the bounties and limitations that mathematics teachers perceive about teacher evaluation in compulsory education. The methodology attended a qualitative paradigm where the informants were 128 mathematics teachers from public school from the regions of the department of Antioquia, Colombia. The teachers taught mathematics at different levels of the Colombian educational system, and to provide information they responded to a survey that inquired about the bounties and limitations of the current evaluation system. The results show that teachers see many more limitations than bounties in the teacher evaluation system despite recognizing its importance. Among the limitations identified by teachers are the subjectivity of the process, little feedback for teachers and little relevance. Within the results it is emphasized that the teacher evaluation system should take into account teacher education, academic production, and the context in which the teacher operates. It is concluded from this study that it is necessary to transform the perspective of the summative evaluation into a formative evaluation that contributes to the improvement of the teaching practice.

Key words: Professional development, Teacher evaluation, Formative evaluation, Teachers' perceptions, Mathematics teachers.

\section{| Introducción}

En los últimos años la evaluación docente ha ganado interés entre investigadores, diseñadores de políticas públicas y profesores, dado que se ha convertido en un proceso clave relacionado con la calidad de los sistemas educativos alrededor del mundo (Coloma, 2010; Escudero, 2019; Tejedor, 2012). Aunque la preocupación por la evaluación docente está presente en muchos países, esta continúa siendo un tema complejo de abordar (Vaillant, 2008). La complejidad radica en múltiples aspectos, entre los cuales se cuentan las formas, los procedimientos y los usos que generan malestar entre los profesores que son sometidos a ella. Específicamente en Colombia, la manera en la que esta usualmente se implementa -como un elemento de control y presión, y reducida a criterios estandarizados (Arizmendi y Saa, 2018) - puede limitar las posibilidades de desarrollo profesional y de mejoramiento continuo, ocultando los verdaderos problemas de la educación.

Adicionalmente, algunas propuestas para evaluar a los profesores sugieren medir el desempeño docente a partir de los resultados de los estudiantes en pruebas académicas (Martínez-Restrepo, Rojas, Ramírez y Pertuz Molina, 2015; Navarro, 2003). Este enfoque es quizá el que ha provocado mayor controversia y debate (Vaillant, 2008), ya que existe poca evidencia empírica acerca del impacto de la evaluación docente en el desempeño de los estudiantes (Isoré, 2009). Tales propuestas son particularmente complejas para los profesores que enseñan matemáticas, dado que, por lo general, es el área con más bajos índices de desempeño de los estudiantes en la mayoría de los sistemas educativos. 
La evaluación docente es actualmente uno de los grandes retos de muchos sistemas educativos (Marchesi, 2009), por lo que debe abordarse con cuidado para que contribuya tanto a la mejora de la profesión como a la de las instituciones educativas (Clipa, 2015). Para lograr tales objetivos, algunas investigaciones (Coloma, 2010; Norris et al., 2017) reconocen la importancia de contar no solo con la participación de los profesores en todo el proceso de evaluación, sino de asumirlos como sujetos de evaluación. Durante el proceso de diseño e implementación de la evaluación docente deben participar varios protagonistas, como actores políticos, administradores, sindicatos, expertos, estudiantes, familia, y los mismos profesores (Valdeavellano, 2008). Es decir, el diseño e implementación de esta debe ser el resultado del conjunto de intereses, concepciones, opiniones y percepciones de dichos actores (Coloma, 2010). Por tanto, debería ser revisada y repensada de forma dialógica por todos sus participantes, incluido el profesor, ya que es él quien debe someterse directamente al proceso evaluativo.

Sin embargo, las opiniones de los evaluados no suelen ser consideradas para diseñar la evaluación (Norris et al., 2017), y, por ende, en muchas ocasiones las políticas nacionales no se corresponden con sus perspectivas (Brown, 2004). Por ello es necesario prestar atención a las percepciones de los profesores sobre la evaluación a la que son sometidos, ya que esta sería inviable sin su participación (Escudero, 2019). Asimismo, la evaluación docente no debería ser un mecanismo para controlar o castigar a los profesores por aspectos que se escapan de su control, tales como los resultados de los estudiantes (Norris et al., 2017).

En este sentido, es fundamental desarrollar un "enfoque integral" (Isoré, 2009) donde se concilien las demandas de calidad educativa con la mejora de las prácticas de enseñanza para el desarrollo profesional, y en el cual los profesores participen en sus propios procesos evaluativos y en el diseño de los sistemas de evaluación (Norris et al., 2017). De esta forma, el interés del presente estudio es analizar las bondades y limitaciones de la evaluación docente, según la perspectiva de un grupo de profesores que enseñan matemáticas en la educación obligatoria ${ }^{1}$ y pública colombiana. Con este estudio se desea aportar reflexiones que permitan revisar el actual sistema de evaluación docente en Colombia.

\section{| Marco teórico}

Para ser coherentes con el objetivo propuesto, el cual está encaminado al análisis de las bondades y limitaciones de la evaluación docente, según la perspectiva de un grupo de profesores colombianos que enseñan matemáticas, el presente apartado se ocupará de desarrollar algunos elementos teóricos con respecto a la evaluación docente. También, se presentarán algunos elementos relacionados con la legislación que rige el actual sistema de evaluación en Colombia para la educación obligatoria.

\footnotetext{
1 Según la Constitución Política de Colombia (1991), la educación obligatoria es responsabilidad del Estado, la sociedad y la familia, desde los 5 hasta los 15 años de edad. Estas edades corresponden a los grados desde preescolar hasta noveno. No obstante, en el Plan Nacional de Desarrollo 2014-2018 se estableció la obligatoriedad de la educación hasta el grado 11. En éste artículo cuando se hable de educación obligatoria se hace referencia al periodo escolar comprendido desde el preescolar hasta el grado 11.
} 


\section{Evaluación docente}

La evaluación sobre el desempeño docente en la educación obligatoria no es una preocupación reciente. Esta preocupación ha sido controversial y ha generado un interés progresivo en distintos países por establecer sistemas evaluativos como un componente esencial de sus políticas educativas. Las demandas de calidad educativa en los diferentes países han llevado al establecimiento de procesos de evaluación, regulados por el Estado, para determinar el desempeño del profesorado (Isoré, 2009). En tal sentido, los sistemas de evaluación pueden adoptar múltiples formas según el contexto y la tradición educativa de cada país (Valencia Rodríguez y Vallejo Cardona, 2015). Del mismo modo, pueden involucrar diferentes actores en el diseño, implementación y formulación de los propósitos bajo los cuales se plantea la evaluación.

De acuerdo con Clipa (2011) e Isoré (2009), la evaluación docente cuenta con dos propósitos principales; por un lado, garantizar que los profesores se desempeñen de la mejor manera posible para mejorar el aprendizaje de los estudiantes, por otro lado, enriquecer continuamente su práctica mediante la identificación de fortalezas y debilidades. Según lo anterior, la evaluación en cuestión posee dos enfoques, sumativo y formativo. El primero se considera como un mecanismo de garantía de calidad (Isoré, 2009), que se ocupa de evaluar las acciones y prácticas de los profesores para mejorar los resultados de los estudiantes. En este sentido, la evaluación docente es concebida como un proceso administrativo que pretende controlar, clasificar, comparar o "medir" (Coloma, 2010) las habilidades y los conocimientos de los profesores. Los resultados de este propósito permiten la toma de decisiones respecto a la contratación, aumento salarial, permanencia y oportunidades de ascenso (Isoré, 2009).

El segundo propósito, el formativo, puede ser orientado hacia la mejora permanente de la práctica docente (Escudero, 2019). Dicho propósito se refiere a una evaluación cualitativa de las prácticas de los profesores, orientada a identificar fortalezas y debilidades en la enseñanza (Clipa, 2011) para ofrecer oportunidades adecuadas de desarrollo profesional en las áreas en las que más se necesita. Es decir, la evaluación bajo esta mirada no es una acción aislada del desarrollo profesional, sino que se asume como parte de un proceso formativo y de mejora que involucre la participación de los docentes para facilitar el reconocimiento de sus conocimientos, la familiarización con la práctica reflexiva, el compromiso, la posibilidad de cambio (Coloma, 2010) y de perfeccionamiento continuo.

Algunos autores (Brown, 2004; Santos, 2012) han reconocido que existe una dicotomía entre la evaluación sumativa y la formativa, que suele generar tensiones a los profesores cuando son evaluados, dado que no se logra distinguir entre ellas. Para Isoré (2009), los aspectos sumativos y formativos de la evaluación son a menudo conflictivos, pero no necesariamente incompatibles, por tanto, se deberían integrar de tal manera que los profesores consideren los procesos evaluativos como una base para mejorar su práctica y como una oportunidad de crecimiento personal y profesional continuo. En tal sentido, la evaluación docente se asume como un proceso sistemático y permanente para apoyar a los profesores en la reflexión sobre su práctica y en la toma de decisiones que impacten positivamente su labor profesional y consecuentemente institucional (Coloma, 2010). 
Diversos investigadores han enfatizado la importancia de estudiar las percepciones de los profesores sobre la evaluación docente (Brown, 2004; Clipa, 2011; 2015; Coloma, 2010; Isoré, 2009; Norris et al., 2017), dado que la forma en que ellos perciben la evaluación se vincula con sus propias experiencias frente a cómo la han vivenciado. Tomando como antecedentes los estudios de Pajares (1992), se comprenden las percepciones del profesor como un concepto que relaciona sus creencias, concepciones, ideologías personales, puntos de vista y prioridades idiosincrásicas, y, por tanto, influyen en sus comportamientos y decisiones (Speer, 2005). En tal sentido, aunque las percepciones no se pueden observar a simple vista, pueden ser inferidas a través de lo que los sujetos dicen.

\section{| Evaluación docente en Colombia}

En Colombia los procesos de evaluación docente, de la educación obligatoria del sector público, están estrechamente asociados con los tipos de contratación del profesor. Actualmente existen dos tipos de contratación: una regida por el Decreto 2277 del año 1979 y otra regulada por el Decreto 1278 del año 2002. Los profesores que ingresaron a la carrera docente antes del 2002 y son regidos por el Decreto 2277 de 1979 están exentos de evaluación y su ascenso en el escalafón depende de los títulos y de la experiencia acumulada que certifiquen (De Zubiría Samper, 2018). Por su parte, los profesores nombrados después del año 2002, por medio del Decreto 1278, están sujetos a dos tipos de evaluación: de desempeño y de ascenso (Vaillant, 2008).

En la legislación colombiana, la evaluación se asume como “un proceso permanente que permite verificar el quehacer profesional de los educadores, identificando fortalezas y aspectos de mejoramiento, mediante la valoración de sus competencias funcionales y comportamentales" (Decreto 3782/2007). Es decir, pareciera que se pretenden integrar los procesos de evaluación formativa y sumativa de manera dialógica y complementaria, pero ¿cómo se concibe y cómo se lleva a cabo? La evaluación docente en Colombia se realiza en tres momentos: período de prueba al ingreso, al finalizar el año escolar y en forma voluntaria para ascender; cada uno de los cuales evalúa a los profesores en distintas etapas de su carrera y con diferentes objetivos (Vaillant, 2008).

El primer momento, que se lleva a cabo durante el periodo de prueba, posee un carácter obligatorio y sirve para evaluar tanto el desempeño como competencias específicas del profesor. Esta primera evaluación ocurre siempre y cuando el profesor haya estado en ejercicio más de cuatro meses; en caso contrario, se deberá esperar hasta la finalización del año escolar siguiente. El segundo momento corresponde a una evaluación anual de desempeño de carácter obligatorio, que es realizada por los administradores de los establecimientos educativos, en las que se establece un plan de metas y mejoramiento para el año posterior. Por último, se encuentra la evaluación de ascenso, que tiene un carácter voluntario y está dirigida a aquellos profesores que pretendan ascender de grado o de nivel salarial en el mismo grado ${ }^{2}$ (Vaillant, 2008). 
Adicionalmente, en el año 2015 se aprobó una nueva propuesta de evaluación docente para ascenso, que incluye un video en lugar de un examen escrito. El vídeo es evaluado por pares y equivale al $80 \%$ de la valoración total, es acompañado de una autoevaluación que corresponde al $10 \%$, y la evaluación de administrativos y estudiantes, que corresponden al restante $10 \%$. Por ser el video el componente que da cuenta del mayor porcentaje en la evaluación de ascenso, se da gran importancia al trabajo que realiza el profesor en el aula de clase (Arizmendi y Saa, 2018). Tanto los resultados de la evaluación anual de desempeño docente como los de ascenso en Colombia son usados para definir el ingreso, permanencia y promoción de los profesores en el sistema educativo obligatorio (Dimaté, Tapiero, González, Rodríguez y Arcila, 2017).

\section{| Metodologia}

El presente estudio analiza las bondades y limitaciones de la evaluación docente según la perspectiva de un grupo de profesores que enseñan matemáticas, en la educación obligatoria, en diferentes regiones del departamento de Antioquia, Colombia. El diseño de la investigación fue de tipo no experimental, transversal y descriptivo (de acuerdo con la clasificación ofrecida por Hernández Sampieri, Fernández Collado y Baptista Lucio, 2014). Se siguió un diseño no experimental con apoyo de una encuesta, la cual es una herramienta útil para la descripción, identificación de tendencias, actitudes u opiniones de una población, mediante el estudio de una muestra de dicha población (Creswell, 2014).

Este estudio se deriva de una investigación macro, cuyo objetivo fue indagar las percepciones de los profesores de matemáticas en ejercicio sobre la evaluación docente. Para dicha investigación macro se diseñó un instrumento con un componente de información demográfica, una escala de percepción (componente cuantitativo) y preguntas abiertas (componente cualitativo). No obstante, en este reporte solo se da cuenta de las respuestas a las preguntas abiertas, debido a que la información restante aún se encuentra en proceso de análisis. El componente cualitativo incluyó tres preguntas abiertas que se realizaron a una muestra de 128 profesores de matemáticas en ejercicio, de diversas instituciones educativas de carácter público, de 22 municipios del departamento de Antioquia.

Para tener representación de los docentes del departamento de Antioquia, cada uno de los miembros del equipo de investigación estableció contacto con instituciones educativas en algunas de las nueve subregiones del departamento e invitó profesores para participar en el estudio. No obstante, la participación fue voluntaria -no recibieron ningún estímulo académico o compensación económica-y los profesores que conformaron la muestra fueron aquellos que atendieron la invitación. Se realizó el proceso de consentimiento informado en el cual se indicó a los participantes los objetivos, riesgos y beneficios del estudio, la participación voluntaria y la no penalidad al no participar o dejar de participar. Se guardó privacidad y confidencialidad con la información ofrecida por los profesores y se garantizó su anonimato de acuerdo con la ley de investigación colombiana (Resolución 8430 de 1993, Ministerio de Salud).

2 En Colombia el escalafón docente establecido en el Decreto Ley 1278 de 2002 tiene tres grados 1, 2 y 3, y en cada grado se encuentran cuatro niveles salariales A, B, C y D. 
Los profesores participantes enseñaban matemáticas en los niveles de preescolar, educación básica y media, y sus contratos laborales correspondían a los dos tipos de vinculación docente considerados en la ley colombiana: Decreto 2277 y Decreto 1278. Los investigadores viajaron a las diferentes regiones del departamento y aplicaron el instrumento en las instituciones educativas cuyos profesores aceptaron participar voluntariamente. El instrumento incluyó las siguientes preguntas abiertas, que indagaban por tres posturas frente a la evaluación: tiene bondades, tiene limitaciones y aspectos por mejorar:

1. ¿Qué bondades tiene el actual sistema de evaluación del profesor?

2. ¿Qué limitaciones tiene el actual sistema de evaluación del profesor?

3. Teniendo en cuenta su experiencia como profesor de matemáticas, indique algunos aspectos que deberían ser considerados en la evaluación del profesor de matemáticas.

Estas preguntas fueron inspiradas en técnicas de evaluación estratégica que permiten elaborar un diagnóstico inicial de un proceso, y basado en él proponer estrategias de intervención. Al indagar sobre las bondades se buscaba estudiar características internas y externas de la evaluación docente que contribuyen positivamente a la mejora del proceso o al desarrollo profesional del profesor evaluado. Al averiguar por las limitaciones se buscaba explorar las características internas y externas de la evaluación docente que no logran hacer aportes al proceso o que por el contrario previenen al profesor de avanzar en su desarrollo profesional. Al indagar por consideraciones adicionales para la evaluación docente se buscaba identificar aquellos aspectos que sistemáticamente han sido ignorados en el proceso, pero que son percibidos como esenciales por los profesores.

El instrumento se validó con dos pruebas piloto con profesores en ejercicio y con discusiones en el equipo de investigación. El pilotaje del instrumento reveló la necesidad de hacer ajustes en la redacción de las preguntas. Se resalta que el instrumento de indagación no pretendía centrar el interés de los participantes en un tipo de evaluación particular (de desempeño o de ascenso), sino en la evaluación en general como un proceso.

Finalmente, el proceso de análisis consiste en darle estructura a una información que carece de ella (Hernández Sampieri et al., 2014). Para el análisis de los resultados obtenidos se sistematizaron las respuestas a cada pregunta en un archivo de Excel. Luego, las respuestas fueron asociadas por categorías en cada pregunta -siguiendo una codificación abierta (Creswell, 2014) - y se agruparon aquellas respuestas que referían a un mismo aspecto. En este proceso de categorización se eliminó la información irrelevante, para que aquella considerada esencial fuera determinando la estructura. En cada categoría se cuantificó la frecuencia simple y se calculó el porcentaje con respecto al total de participantes que respondieron en cada pregunta. Los resultados de dicho proceso se presentan en el siguiente apartado. 


\section{Resultados}

Los resultados se agruparon en tres apartados: bondades de la evaluación docente, limitaciones de la evaluación docente y consideraciones adicionales en la evaluación docente.

\section{Bondades de la evaluación docente}

A la pregunta que indaga sobre las bondades del actual sistema de evaluación docente en Colombia, solo 105 participantes respondieron y ofrecieron diversidad de respuestas. Dichas respuestas se organizaron en frecuencias simples y se presentan de la más frecuente a la menos frecuente en la tabla 1.

\section{Tabla 1}

Bondades de la evaluación docente según la perspectiva de los profesores

\begin{tabular}{lcc}
\hline \multicolumn{1}{c}{ Respuesta } & Frecuencia & Porcentaje \\
\hline Ninguna & 48 & 45,7 \\
Permite el mejoramiento y cualificación del profesor & 15 & 14,2 \\
Permite valorar lo que hace el profesor & 8 & 7,6 \\
Permite la reflexión & 7 & 6,6 \\
Permite el autoconocimiento & 5 & 4,7 \\
Permite la participación del profesor & 4 & 3,8 \\
Permite ascender en el escalafón & 4 & 3,8 \\
Permite la retroalimentación & 3 & 2,8 \\
Permite el mejoramiento económico & 2 & 1,9 \\
No sabe & 2 & 1,9 \\
Permite al profesor innovar & 2 & 1,9 \\
Permite estabilidad laboral & 1 & 0,9 \\
Permite la participación de otros (coevaluación) & 1 & 0,9 \\
Permite mirar diferentes aspectos & 1 & 0,9 \\
Permite el mejoramiento en la calidad educativa & 1 & 0,9 \\
Exige actualización & 1 & 0,9 \\
\hline
\end{tabular}

Nota: elaboración propia 
Como se aprecia en la tabla 1, la respuesta más frecuente de los profesores participantes fue que la evaluación docente no tenía ninguna bondad. Algunos justificaron su respuesta en los siguientes términos: "Ninguna [bondad] porque finalmente no tenemos o no recibimos una retroalimentación de fortalezas y debilidades" (Participante 41), "Ninguna [bondad], me genera estrés, temor, inseguridades, empiezo el proceso, pero no lo termino (no me gusta)" (Participante 43), "[Ninguna], no se ha tenido en cuenta el maestro y sus deficiencias para la construcción de la misma" (Participante 36), y "Considero que [la evaluación] no tiene bondades, debido a que es a final de año y en si no se hace un seguimiento sobre esto, es por cumplir" (Participante 73).

La falta de bondades de la evaluación docente, señalada por un alto número de los profesores, incluyó justificaciones relacionadas con sentimientos poco agradables frente a esta (temor, estrés, inseguridad), con la poca o ninguna retroalimentación que se les ofrece, y con la poca o nula participación en la construcción de las evaluaciones, entre otros. La tabla 1 también muestra que, aunque los profesores señalaron algunas bondades de la evaluación -oportunidad de mejoramiento y la cualificación (14,2\%) ("[La evaluación] sirve para mejorar el quehacer pedagógico (Participante 121)), herramienta para valorar el quehacer del profesor (7,6\%) (“Evalúa el quehacer del profesor" (Participante 19)), y para promover la reflexión (6,6\%) ("[La evaluación] pone en reflexión al docente con su quehacer" (Participante 4))-, llama la atención que el 45,7\% de los profesores no reconocen una sola bondad en el sistema de evaluación. Es decir, aunque algunos valoran la importancia del proceso evaluativo como oportunidad formativa, otros profesores no ven bondades en él y poseen una percepción poco favorable de la evaluación docente.

\section{| Limitaciones de la evaluación docente}

En relación con las limitaciones señaladas por los participantes sobre el sistema actual de evaluación docente, solo 104 profesores ofrecieron información y expresaron opiniones en una multiplicidad de frentes. La tabla 2 presenta los resultados organizados del más frecuente al menos frecuente. 


\section{Tabla 2}

Limitaciones de la evaluación docente según la perspectiva de los profesores

\begin{tabular}{lcc}
\hline \multicolumn{1}{c}{ Respuesta } & Frecuencia & Porcentaje \\
\hline Mide muchas dimensiones (no mide lo que debe medir) & 14 & 13,5 \\
Es instrumental (se centra más en evidencias que en & 9 & 8,6 \\
procesos) & 8 & 7,7 \\
Es subjetiva & 8 & 7,7 \\
No ofrece retroalimentación & 7 & 6,7 \\
Es un medio de control y sanción & 7 & 6,7 \\
No permite ascender & 7 & 6,7 \\
No es pertinente & 6 & 5,7 \\
Presupuesto insuficiente & 6 & 5,7 \\
No tiene en cuenta la capacitación y actualización de los & & \\
profesores & 5 & 4,8 \\
Es excluyente & 4 & 3,8 \\
Es descontextualizada & 3 & 2,9 \\
No tiene en cuenta informantes importantes de la & & \\
comunidad educativa & 3 & 2,9 \\
Es injusta & 3 & 2,9 \\
No genera transformación & 3 & 2,9 \\
No discrimina (todos pasan) & 3 & 2,9 \\
Es acomodada a politicas de Estado & 2 & 1,9 \\
No tiene en cuenta al profesor & 2 & 1,9 \\
Ninguna & 1 & 0,9 \\
No tiene en cuenta la experiencia del profesor & 1 & 0,9 \\
No es clara & 1 & 0,9 \\
No es consistente & 1 & 0,9 \\
Puede existir acoso laboral &
\end{tabular}

Nota: elaboración propia

De acuerdo con la tabla 2, un alto porcentaje de participantes indicaron como limitación del sistema de evaluación docente la pretensión de medir muchas dimensiones (13,5\%) y no enfocarse en lo que en realidad debería ser evaluado. Con respecto a esta limitación, los profesores dieron las siguientes respuestas: "[La evaluación] tiene demasiados criterios a evaluar" (Participante 124), "No hay profundización en los criterios a evaluar" (Participante 125), y "Los aspectos que maneja la evaluación son demasiados" (Participante 65). Esto sugiere que los profesores perciben el sistema de evaluación docente como ambicioso. 
Otra de las limitaciones que apareció con frecuencia en las respuestas de los participantes, con respecto al actual sistema de evaluación docente, fue la instrumentalización de la evaluación. Algunas de las apreciaciones dadas por los profesores frente a dicho aspecto fueron las siguientes: "[La evaluación] se preocupa más de las evidencias que de los procesos" (Participante 6), "[La evaluación] se ha convertido en una práctica meramente instrumental" (Participante 41), y "[La evaluación] no evidencia el verdadero trabajo del docente, es [un proceso] muy técnico" (Participante 89). Es decir, los profesores expresaron que la evaluación da más importancia a los instrumentos para aportar evidencias o para medir resultados, y menos a los procesos formativos.

Los participantes también señalaron como limitación la percepción que se tiene de ella como un mecanismo de control y sanción. A este respecto surgieron expresiones tales como: "[La evaluación] no genera cambios, solo es un medio de control" (Participante 22), "Es una evaluación de control y presión laboral" (Participante 102), "[La evaluación] no es diagnóstica, es sancionatoria" (Participante 32), y "[La evaluación] es una herramienta de control y no de mejoramiento" (Participante 101). En tal sentido, los profesores insistieron en señalar que la evaluación docente se reduce a prácticas de control, sanción, y presión que poco aportan a la transformación y mejoramiento de la acción del profesor en el aula y en la institución.

Otras limitaciones del sistema de evaluación docente señaladas por los participantes están relacionadas con: la subjetividad del proceso (7,7\%), la poca retroalimentación para los docentes $(7,7 \%)$, y la poca pertinencia (6,7\%) (ver tabla 2). Con base en tales indicadores, los profesores ofrecieron argumentos como: "[La evaluación] es más subjetiva que objetiva porque no refleja mucho la práctica del aula" (Participante 121), "[La evaluación] no entrega resultados con el fin de mejorar" (Participante 5), y "[La evaluación] no es constante ni oportuna" (Participante 119). Las diferentes limitaciones aludidas por los profesores reafirman una vez más la imagen poco favorable que poseen de la evaluación docente y la manera como esta es implementada en Colombia.

\section{| Consideraciones adicionales en la evaluación docente}

Con esta pregunta se querían indagar aspectos que los profesores participantes valoraran como esenciales en el proceso de evaluación docente del profesor que enseña matemáticas, y que no son considerados en el actual sistema. Infortunadamente, solo 47 profesores proporcionaron información para esta pregunta. La tabla 3 muestra las respuestas de los profesores participantes que ofrecieron información y están organizadas de la más frecuente a la menos frecuente. 
Tabla 3

Consideraciones adicionales para la evaluación docente según la perspectiva de los profesores

\begin{tabular}{lcc}
\hline \multicolumn{1}{c}{ Respuesta } & Frecuencia & Porcentaje \\
\hline Tener en cuenta la formación académica de los profesores & 10 & 21,2 \\
Que se evalúe lo que el profesor hace en el aula & 6 & 12,7 \\
Tener en cuenta los aprendizajes de los estudiantes & 5 & 10,6 \\
Considerar la producción académica (material didáctico, & 3 & 6,3 \\
artículos, ponencias) & 3 & \\
Tener en cuenta la experiencia docente & 3 & 6,3 \\
Privilegiar los procesos por encima de las evidencias & 3 & 6,3 \\
Tener en cuenta el acompañamiento integral que el profesor & 3 & 6,3 \\
hace al estudiante & 3 & \\
Tener en cuenta el contexto & 2 & 6,3 \\
Gestión humana & 2 & 4,2 \\
Que la evaluación sirva para mejorar & 1 & 4,2 \\
Debe ser una evaluación integral & 1 & 2,1 \\
Debe ser una evaluación basada en un sistema de estímulos & 1 & 2,1 \\
Brindar acompañamiento a los profesores & 1 & 2,1 \\
Proyección comunitaria & 1 & 2,1 \\
Está bien diseñada & 1 & 2,1 \\
Tiene demasiados aspectos para considerar más & 1 & 2,1 \\
Ninguna & 2,1 \\
\hline
\end{tabular}

Nota: elaboración propia

Entre los aspectos que los profesores consideran como componentes esenciales de la evaluación, parece existir un consenso en relación con la valoración de las certificaciones académicas (21,2\%). Al respecto los profesores expresaron: “De acuerdo con las especializaciones tenerlas en cuenta para la evaluación” (Participante 76), "De acuerdo con sus especialidades tener presente el aumento salarial" (Participante 77), "[Que se tengan en cuenta] cursos o espacios de formación en los que participe el docente" (Participante 55), "Su nivel de formación y capacitación, pero no por pruebas externas, evaluaciones para poder que sean reconocidos los títulos. Sino ofrecer más incentivos para el que se forma y se preocupa por mejorar académica y profesionalmente" (Participante 103). Esto sugiere que los profesores reclaman que las certificaciones académicas sean tenidas en cuenta cuando son evaluados.

Otro aspecto que los profesores resaltaron refiere a tener en cuenta lo que en efecto hace el profesor. Algunos participantes hicieron alusión a ello en los siguientes términos: "[La evalua- 
ción debería tener en cuenta] la planeación, las diversas estrategias, planes de mejoramiento y formas de evaluar [de los profesores]" (Participante 128), "[La evaluación debería tener en cuenta] las estrategias metodológicas y evaluativas que [los profesores] implementan en sus clases" (Participante 74), "se debería de evaluar la adaptación del currículo a las dificultades [de los estudiantes]" (Participante 75), y "[La evaluación debería tener en cuenta] la proyección comunitaria" (Participante 106). Esto incluye preparaciones de clase, proyección a la comunidad, evaluación a sus estudiantes, uso de recursos didácticos, adaptaciones del currículo a las dificultades de los estudiantes.

Los profesores también consideran que el sistema de evaluación docente debería incluir elementos como la experiencia docente, la producción académica del profesor (materiales didácticos, ponencias, artículos), y el contexto particular en el que se lleva a cabo la evaluación. Además, los participantes señalaron que la evaluación docente debería asumirse como un proceso integral, donde se reconozca al profesor como un ser y no como un "objeto" de evaluación, y que ofrezca acompañamiento al docente en su proceso de formación y mejoramiento continuo.

\section{| Discusión}

A continuación, se presenta una discusión relacionada con las bondades, limitaciones y consideraciones adicionales que los participantes señalaron, respecto a la evaluación docente de los profesores que enseñan matemáticas. Aquí se discuten los resultados y algunas implicaciones para el sistema de evaluación docente.

Las bondades de la evaluación docente señaladas por los participantes coinciden con los propósitos formativos de la evaluación como un proceso permanente, ya que esta se reconoce como una posibilidad para mejorar profesionalmente (Escudero, 2019) y como una oportunidad para reflexionar sobre su propia actividad que, potencialmente, podría orientar la toma de decisiones y la cualificación de la labor profesional (Coloma, 2010). No obstante, la percepción tan poco favorable de dicho proceso en Colombia parece estar promovida por las formas como se implementa y por los usos que se le da. En muchas ocasiones el sistema de evaluación no ayuda a mejorar la práctica docente, no contribuye al desarrollo profesional ni a la mejora salarial (De Zubiría Samper, 2018). La evaluación docente se reduce a un asunto de selección y clasificación de los profesores.

La falta de bondad que manifiestan los profesores en el sistema de evaluación docente podría estar relacionada con el uso instrumental (señalado por De Alba, Díaz Barriga y Viesca, 1984), con el que también se asume dicha evaluación en Colombia. Este uso instrumental privilegia la aplicación de técnicas que genera y aglutina datos, pero que oculta la reflexión conceptual sobre la evaluación. Esta visión de la evaluación, según De Alba et al. (1984), responde a la noción del "manejo científico del trabajo", desarrollado por Frederick W. Taylor al 
principio del siglo XX en el contexto de la administración, y que a su vez responde a las exigencias de eficiencia del capital. Dicha noción instrumental de la evaluación busca definir un lugar para el individuo en la escala de producción.

Es el profesor a quien se busca certificar o acreditar en la profesión docente, determinar sus capacidades, medir, controlar; pero de este proceso se beneficia poco, dado que tiene pocas posibilidades de retroalimentación y de mejoramiento a partir de los resultados de la evaluación. Además, no hay posibilidad de sentar una posición crítica respecto al proceso evaluativo.

El problema de la técnica ha ocupado un lugar privilegiado que tiende a relegar los aspectos académicos, políticos e ideológicos, subordinándolos a una racionalidad técnica de orden científico que toma como punto de partida el estado de las cosas existente. (De Alba et al., 1984, p. 118).

La visión instrumentalista de la evaluación docente puede identificar al profesor promedio, acreditar a unos y desacreditar a otros; pero termina ignorando problemas fundamentales de la educación como la desfinanciación, la falta de cobertura y la carencia de programas para la formación continua de profesores. La formulación de un juicio de valor mediante la evaluación docente debe tomar en cuenta los diferentes espacios de autonomía entre las relaciones significativas, entre la sociedad y sus instituciones, pero la mirada instrumental de la evaluación ignora esta relación dialéctica.

Este aspecto llama la atención puesto que varios académicos han señalado que cuando la evaluación se centra en el aspecto técnico reduce cualquier posibilidad de reflexión (De Alba et al., 1984; Valencia Rodríguez y Vallejo Cardona, 2015). Así, los aspectos académicos, políticos e ideológicos que circundan los procesos de evaluación quedan subordinados a lo técnico. Este no es un hallazgo simple si se tiene en cuenta que la noción de evaluación en el mundo surge a la par de los procesos de industrialización, y, desde su aparición, esta noción ha estado estrechamente ligada con las exigencias capitalistas de eficiencia.

Por otro lado, asumir la evaluación como un mecanismo de sanción y de control se relaciona con la concepción técnica de la misma, que es coherente con discusiones sugeridas en la literatura, en la cual se atribuye a la evaluación la connotación de mecanismo de control y auditoría en búsqueda de la eficiencia y la eficacia (De Alba et al., 1984). El control es una de las etapas del proceso administrativo que nace también con la aparición de las industrias, con lo cual se sugiere que la evaluación docente surge al considerar las escuelas como empresas.

Con respecto a la falta de objetividad reclamada por los profesores participantes en el actual sistema de evaluación docente, se reconoce que la objetividad es lo que se espera en cualquier estudio con pretensiones científicas; sin embargo, esta implica grandes dificultades, en especial cuando su propósito consiste en emitir una valoración (Tiana, 2009), como suele suceder en la evaluación docente. Es por ello, que el asunto de la subjetividad genera tensiones entre los profesores al interior de los procesos evaluativos, y se convierte en una de las razones por las 
cuales los profesores consideran que la evaluación ofrece poca retroalimentación y es poco pertinente para sus procesos de mejoramiento (Coloma, 2010; Tejedor, 2012).

La subjetividad parece ser un elemento connatural al proceso de evaluación docente, pues a pesar de utilizar diversos instrumentos y tipos de información, la valoración realizada por los agentes encargados es la que se asume como definitiva (Díaz, 2014; Martínez-Restrepo et al., 2015). En este sentido, surge el cuestionamiento sobre la objetividad del proceso. Muchas veces los encargados de llevar a cabo la evaluación docente ponen en este ejercicio sus propias creencias, experiencias y sus convicciones, dejando de lado los propósitos explícitos y los elementos objetivos de la evaluación (Díaz, 2014). Así, por ejemplo, un agente encargado de la evaluación docente que valore el orden le dará mucha más importancia a la estructura del salón de clase y a la metódica disposición de los estudiantes que al conocimiento matemático que allí tenga lugar. Aunque es imposible eliminar por completo la subjetividad de la evaluación - pues esta es implementada por seres humanos (Tiana, 2009) -, es necesario disminuirla para dotar al proceso de un carácter objetivo y justo (Coloma, 2010).

Un argumento que puede explicar las numerosas limitaciones que los profesores participantes señalaron del actual sistema de evaluación docente tiene que ver con la forma en que experimentan y viven este proceso. La observación empírica de los procesos de evaluación revela que "están única y exclusivamente orientados a la selección social" (Mora, 2005), lo cual los aleja de su sentido fundamental, relacionado con su función didáctica y pedagógica, distorsionando sus principios formativos (Díaz, 2014). En consecuencia, muchas veces los profesores consideran que los propósitos formativos de la evaluación docente son declarados en el papel, pero dificilmente se dan en el plano real (Díaz, 2014), convirtiendo la evaluación en un proceso informal y puramente protocolario.

Las limitaciones de la evaluación docente señaladas por los participantes sugieren un cuestionamiento frente a la forma como se orienta actualmente este proceso en Colombia. Se hace evidente la necesidad de trascender la concepción de la evaluación como un medio técnico de gestión administrativa que pretende controlar, comparar, clasificar y medir a los docentes (Coloma, 2010; Tejedor, 2012), y les da mayor importancia a los propósitos sumativos que a los formativos.

Con base en las consideraciones adicionales para tener en cuenta en el actual sistema de evaluación docente, señalados por los profesores, es importante resaltar el reclamo de los participantes para que se tenga en cuenta lo que en efecto hace el profesor. Este llamado de los profesores es interesante y parece sugerir que muchas de las tareas asociadas con la labor del profesor quedan ocultas en el actual sistema de evaluación docente (Díaz, 2014), ya que este continúa centrándose más en resultados que en los procesos y las acciones del profesor (Valencia Rodríguez y Vallejo Cardona, 2015).

Adicional a lo anterior, los profesores hacen un llamado para que la evaluación docente se lleve a cabo de manera integral. Para Arizmendi y Saa (2018), es necesario que la evaluación sea un proceso integral y sistémico, que no sólo se quede en medir los conocimientos de los profesores sobre ciertas disciplinas, sino que ofrezca acompañamiento y retroalimentación a los docentes, y permita tomar decisiones para la mejora educativa. Estas declaraciones sugieren 
un reclamo de los profesores para que se privilegien los procesos por encima de las evidencias, y para que la evaluación sea vista como una herramienta para mejorar el desempeño profesional.

Los llamados y reclamos realizados por los profesores frente a la manera en que son evaluados actualmente en Colombia se convierten en un insumo importante para repensar y resignificar el actual sistema de evaluación docente. Conocer las percepciones de los docentes frente a dicho proceso es un punto de partida para reconocer la importancia de incluir, en los diseños de las evaluaciones, a quienes son evaluados. Además, los resultados encontrados aportan reflexiones basadas en evidencia empírica, que sugieren reorganizar el actual sistema de evaluación docente en Colombia, para que este valioso y crucial proceso cumpla su función formativa y de mejoramiento de la labor del profesor.

Permitir que los profesores expresen sus posiciones con respecto a la evaluación docente, reconociendo sus bondades, limitaciones y aspectos adicionales que se deberían considerar, es dar la voz a aquellos que por mucho tiempo han sido silenciados, pero que deben sufrir las consecuencias de lo que perciben como una evaluación poco formativa. Es aquí donde está el valor agregado del presente estudio, al intentar mostrar que los profesores, como sujetos protagonistas de la evaluación, tienen mucho que decir y que aportar para la transformación y reorganización de los procesos de evaluación docente.

\section{| Conclusiones e implicaciones}

El propósito de este artículo fue analizar las bondades y limitaciones de la evaluación docente, según la perspectiva de un grupo de profesores que enseñan matemáticas en la educación obligatoria en Colombia. A partir de los análisis presentados se concluyen algunas consecuencias teóricas y prácticas y algunas implicaciones.

Los resultados demuestran que, aunque los profesores reconocen la importancia de la evaluación docente como una herramienta para el mejoramiento continuo, le atribuyen más limitaciones que bondades. Entre las limitaciones, reiteradamente señaladas, se encuentran lo ambicioso del sistema, lo subjetivo del proceso y el carácter técnico-instrumental que desdibuja cualquier posibilidad de mejoramiento. La evaluación docente es mucho más que un asunto técnico y debe asumirse como un hacer social que ayude a entender la acción del profesor de matemáticas en el aula, identificar fortalezas, emprender programas de mejoramiento y dar sentido a los fenómenos educativos.

Los resultados revelan que el sistema actual de evaluación docente no promueve el desarrollo profesional de quienes son sometidos a dicho proceso. Es vital, entonces, transfor- 
mar esa visión técnica para convertirla en un medio formativo donde se tome en consideración al docente como un profesional reflexivo y crítico, capaz de aportar a la transformación de los procesos evaluativos. Esta nueva concepción de la evaluación debe tener en cuenta las experiencias del profesor, su conocimiento y los contextos de desempeño profesional para que cumpla su función formativa. Adicionalmente, es necesario establecer una relación dialógica entre la evaluación del desempeño docente y las oportunidades de desarrollo profesional continuo como un elemento esencial para mejorar la práctica del profesor.

Según las declaraciones de los decretos colombianos que regulan la evaluación, se pretende que esta sea medida y conocida objetivamente, pero a pesar de la subjetividad inherente a la evaluación, es posible diseñar instrumentos que cumplan con los objetivos de mejora profesional. Sin embargo, esta declaración contenida en las normas regulatorias de la evaluación, no se cumple. De acuerdo con las percepciones de los profesores, la evaluación debería estar basada en mejor evidencia objetiva, involucrar a las audiencias interesadas -profesores, directivos, expertos externos, comunidad educativa local-, y cumplir con sus propósitos formativos y sumativos de manera integral.

Los profesores insisten, a través de sus respuestas, en que la evaluación debe ser un proceso formativo, integral y sistemático que realmente aporte a la mejora de la práctica futura, y donde cada profesor tenga oportunidades de crecimiento profesional y personal, más que obtener una medida cuantitativa de su desempeño. Una implicación práctica de estos resultados es la necesidad de incluir otras variables en el proceso de evaluación docente para que cumpla con el objetivo de ayudar al mejoramiento de la acción del profesor en el aula y en consecuencia de la calidad del servicio educativo.

Los resultados encontrados constituyen un desafio para el actual sistema de evaluación docente en Colombia, el cual desconoce al profesor como un participante crucial para el diseño de los procesos evaluativos. Por ello, es importante hacer un llamado a vincular a los profesores en dichos diseños, ya que es necesario conocer más sobre cómo ellos perciben la evaluación docente; principalmente porque ella es un componente fundamental para el diseño de políticas públicas educativas y para la toma de decisiones.

La evidencia empírica proporcionada mediante este estudio es un punto de partida para justificar la importancia de vincular a los profesores que enseñan matemáticas en el diseño e implementación de la evaluación docente, que debe convertirse en un proceso incluyente en el cual se dé voz a todos los sujetos que participan en ella. El proceso evaluativo se enriquece mediante la participación de diversos actores, pero el profesor cobra protagonismo al ser quien se somete directamente a él y por ello es el mejor informante para identificar fortalezas y debilidades en los sistemas de evaluación, tal y como se evidenció en este estudio.

El valor de esta investigación refiere a varios aspectos sobre la evaluación docente: el primero, considera la valoración por parte de los profesores quienes están sometidos a ella; el segundo, refiere a las limitaciones que los profesores identifican, y el tercero refiere a aspectos que deberian ser considerados. Estos aspectos identificados por los profesores deberian ser considerados por los entes estatales gestores de la evaluación docente en la educación obligatoria. 


\section{| Agradecimientos}

Esta investigación fue financiada por Colciencias, contrato 438-2017. Se agradece de manera especial a los profesores participantes por sus valiosos aportes.

\section{| Referencias}

Arizmendi, A. y Saa, B. (2018). La evaluación docente en Colombia [Teacher evaluation in Colombia]. Revista Educación, Política y Sociedad, 2(3), 60-75.

Brown, G. T. (2004). Teachers' conceptions of assessment: implications for policy and professional development. Assessment in Education, 11(3), 301-318.

Clipa, O. (2011). Teacher perceptions on teacher evaluation: the purpose and the assessors within the assessment process. Procedia - Social and Behavioral Sciences, 29, 158-163.

Clipa, O. (2015). Roles and Strategies of Teacher Evaluation: Teachers' Perceptions. Procedia Social and Behavioral Sciences, (180), 916-923.

Coloma, C. R. (2010). Estudio comprensivo sobre la evaluación del docente. Revista Iberoamericana de Evaluación Educativa, 3(1), 61-76.

Colombia. (1991). Constitución Política de Colombia. Bogotá, Colombia.

Creswell, J. W. (2014). Research design: qualitative, quantitative and mixed methods approaches ( $4^{\circ}$ ed.). Los Ángeles: SAGE.

De Alba, A., Díaz Barriga, A. y Viesca, M. (1984). Evaluación: análisis de una noción. Revista Mexicana de Sociología, 46(1), 175-204.

De Zubiría Samper, J (2018). La evaluación docente en Colombia frente a América Latina.

Recuperado de

https:/ / www.magisterio.com.co/articulo/la-evaluacion-docente-en-colombia-frente-americalatina

Departamento Nacional de Planeación -DNP-. (2015). Plan Nacional de Desarrollo 2014-2018. Bogotá, Colombia. Recuperado de https://colaboracion.dnp.gov.co/CDT/PND/PND\%202014-2018\%20Tomo\%201\%20internet.pdf

Díaz, E. J. (2014). Características, principios y fines de la evaluación del desempeño docente. Revista Praxis, (10), 8-20. 
Dimaté, C., Tapiero, O., González, C. I., Rodríguez, R. y Arcila, M. A. (2017). La evaluación del desempeño docente [Teaching Performance Assessment]. Folios, (46), 83-95.

Escudero, T. (2019). Evaluación del profesorado como camino directo hacia la mejora de la calidad educativa. Revista de Investigación Educativa, 37(1), 15-37.

Hernández Sampieri, R., Fernández Collado, C. y Baptista Lucio, P. (2014). Metodología de la investigación ( $6^{\circ}$ ed.). México: McGraw Hill.

Isoré, M. (2009). Teacher Evaluation: Current Practices in OECD Countries and a Literature Review. OECD Education Working Papers, (23), 1-48. http://dx.doi.org/10.1787/223283631428

Marchesi, Á. (2009). Preámbulo. En E. Martín y F. Martínez (Eds.), Avances y desafios en la evaluación educativa (pp. 7-10). Madrid, España: OEI - Fundación Santillana.

Martínez-Restrepo, S., Rojas, M., Ramírez, J. M. y Pertuz Molina, M. C. (2015). ¿Necesita la educación colombiana un sistema de evaluación docente? ¿Cuál y para qué? Alianza Compartir Fedesarrollo. Recuperado de https://compartirpalabramaestra.org/documentos/fedesarrollo_compartir/necesita-la\%20ed ucacion-\%20colombiana-un-sistema-de-evaluacion-docente-cual-y-para-que.pdf

Ministerio de Salud. (4 de octubre de 1993). Resolución 8430, por la cual se establecen las normas científicas, técnicas y administrativas para la investigación en salud.

Mora, D. (Coord.). (2005). Didáctica crítica, educación crítica de las matemáticas y etnomatemática: perspectivas para la transformación de la educación matemática en América Latina. La Paz, Bolivia: Campo Iris.

Navarro, J. C. (2003). La evaluación y las actitudes de los docentes frente a ella: dificultades y alternativas de políticas. Buenos Aires: IIPE-UNESCO.

Norris, J., Van der Mars, H., Kulinna, P., Amrein-Beardsley, A., Kwon, J. \& Hodges, M. (2017). Physical Education Teacher Perceptions of Teacher Evaluation. The Physical Educator, 74, 41-62.

Pajares, F. (1992). Teachers囚 Beliefs and Educational Research: Cleaning up a Messy Construct. Review of Educational Research, 62(3) 307-332.

Presidencia de la República de Colombia. (14 de septiembre de 1979). Decreto 2277, por el cual se adoptan normas sobre el ejercicio de la profesión docente.

Presidencia de la República de Colombia. (19 de junio de 2002). Decreto 1278, por el cual se expide el Estatuto de Profesionalización Docente.

Presidencia de la República de Colombia. (7 de octubre 2007). Decreto 3782, por el cual se reglamenta la evaluación anual de desempeño laboral de los servidores públicos docentes y directivos docentes que se rigen por el Decreto Ley 1278 de 2002. 
Santos, A. (2012). Evaluación Docente. Educación Química, 23(2), 200-204.

Speer, N. (2005). Issues of methods and theory in the study of mathematics teachers' professed and attributed beliefs. Educational Studies in Mathematics, 58(3) 361-391.

Tejedor, F. (2012). Evaluación del desempeño docente. Revista Iberoamericana de Evaluación Educativa, 5(1), 319-327.

Tiana, A. (2009). Evaluación y cambio educativo: los debates actuales sobre las ventajas y los riesgos de la evaluación. En E. Martín y F. Martínez (Eds.), Avances y desafios en la evaluación educativa (pp. 17-26). Madrid, España: OEI - Fundación Santillana.

Vaillant, D. (2008). Algunos marcos referenciales para la evaluación del desempeño docente en américa latina. Revista Iberoamericana de Evaluación Educativa, 1(2), 8-22.

Valdeavellano, R. (2008). La evaluación docente no es un asunto esdrújulo: la importancia de los modelos comprensivos. En D. Vaillant y S. Cuba (Eds.), Profesión docente en latinoamérica: una agenda pendiente y cuatro retos emergentes (pp. 83-87). Lima, Perú: Tarea Asociación de Publicaciones Educativas.

Valencia Rodríguez, W. A. y Vallejo Cardona, J. D. (2015). La evaluación educativa: más que una acción, una cuestión ética. Revista Virtual Universidad Católica del Norte, (45), 210-234. Recuperado de http://revistavirtual.ucn.edu.co/index.php/RevistaUCN/article/view/668/1199 